\title{
Determination of the concentration of cathepsin B by SPRI biosensor in children with appendicitis, and its correlation with proteasomes
}

\author{
Ewa Matuszczak ${ }^{1, A-D}$, Marta Komarowska, , , Marzena Tylicka 2,B,C, Wojciech Dębek1, ${ }^{1, F}$, \\ Ewa Gorodkiewicz ${ }^{3, F}$, Anna Tokarzewicz ${ }^{3, B}$, Anna Sankiewicz ${ }^{3, B}$, Adam Hermanowicz, ${ }^{1, F}$ \\ ${ }^{1}$ Department of Pediatric Surgery, Medical University of Bialystok, Poland \\ 2 Department of Biophysics, Medical University of Bialystok, Poland \\ ${ }^{3}$ Department of Electrochemistry, Institute of Chemistry, University of Bialystok, Poland \\ A - research concept and design; $\mathrm{B}$ - collection and/or assembly of data; $\mathrm{C}$ - data analysis and interpretation; \\ $D$ - writing the article; $E$ - critical revision of the article; $F$ - final approval of the article
}

Address for correspondence

Ewa Matuszczak

E-mail: ewamat@tlen.pl

Funding sources

None declared

Conflict of interest

None declared

Received on December 31, 2016

Reviewed on March 6, 2017

Accepted on April 27, 2017

DOI

10.17219/acem/70811

Copyright

Copyright by Author(s)

This is an article distributed under the terms of the

Creative Commons Attribution Non-Commercial License

(http://creativecommons.org/licenses/by-nc-nd/4.0/)

\begin{abstract}
Background. Cathepsin B (CatB) belongs to a family of lysosomal cysteine proteases and plays an important role in intracellular proteolysis.

Objectives. The concentration of CatB and 205 proteasome was evaluated in the serum of children with appendicitis, before and after surgery, on a basis of an innovative method for determining biomolecules concentration - surface plasmon resonance imaging (SPRI) biosensor.
\end{abstract}

Material and methods. Forty-two children with acute appendicitis, who were treated at the Department of Pediatric Surgery (Medical University of Bialystok, Poland), were randomly included into the study (age: 5-17 years, mean age: $11.5 \pm 1$ year). There were 15 girls and 27 boys in the study group. Eighteen healthy, age-matched subjects, admitted for planned surgeries, served as controls. Exclusion criteria were the following: severe preexisting infections, immunological or cardiovascular diseases that required longterm medication, and complicated cases of appendicitis with perforation of the appendix and/or peritonitis.

Results. The CatB concentrations in the blood plasma of patients with acute appendicitis were elevated before surgery, they were the highest $24 \mathrm{~h}$ after surgery, and were above the range of concentrations measured in controls; the difference was statistically significant. The CatB concentration measured $72 \mathrm{~h}$ after the operation was decreased, but still did not reach the normal range when compared with the concentration measured in controls $(p<0.05)$.

Conclusions. Cathepsin B concentration may reflect the metabolic response to acute state of inflammation, surgical intervention in the abdominal cavity and the process of gradual ebbing of the inflammation. The method of operation - classic open appendectomy or laparoscopic appendectomy - does not influence the general trend in the CatB concentration in children with appendicitis. There is a strong positive correlation between the CatB and 205 proteasome concentrations $24 \mathrm{~h}$ after surgery. The SPRI method can be successfully used for determining the concentration of active forms of enzymes presented in lysosomes in the diagnosis of inflammatory conditions in the abdominal cavity.

Key words: appendicitis, inflammation, proteasomes, cathepsin B, surface plasmon resonance imaging biosensor 


\section{Introduction}

Cathepsins are a family of acidic endopeptidases, the activity of which is restricted to the lysosomal compartment under physiological conditions. ${ }^{1}$ Cathepsins take part in protein turnover by degrading unneeded proteins into amino acids. ${ }^{1}$ Various pathologic stresses, such as global cerebral ischemia, induce the release of cathepsins into the cytoplasm, where they perform their proteolytic function and can promote direct cell degradation. ${ }^{1-3}$ Cathep$\sin B$ (CatB) directly activates caspase-11 and/or caspase-1 pathways, e.g., in focal cerebral ischemia, and confirms that apoptosis, necrosis and autophagia are interrelated mechanisms that can act in synergy and lead to cell death in acute pathologic conditions. ${ }^{1}$ Cathepsin $\mathrm{B}$ is also responsible for nuclear factor kappa-light-chain-enhancer of activated $B$ cells (NF- $\mathrm{kB}$ ) activation through autophagy degradation of inhibitor of $\kappa \mathrm{B} \alpha(\mathrm{I} \kappa \mathrm{B} \alpha)$ in microglia and macrophages. ${ }^{4}$ The inhibition of CatB can rescue cells from tumor necrosis factor (TNF)-induced apoptosis. ${ }^{5}$ The inhibition of cathepsins leads to significant neuroprotection, because treatment with a specific CatB inhibitor significantly reduces infarct volumes. ${ }^{1}$

Elevated levels of CatB in fibroblasts are typically observed in many chronic inflammatory diseases, including rheumatoid arthritis as well as periodontitis, because CatB promotes inflammation involved in the production of mature interleukin $1 \beta$ (IL-1 $\beta) .{ }^{4,6-8}$ Interleukin $1 \beta$ is a potent pro-inflammatory cytokine that is crucial for host-defense responses to infection and injury. ${ }^{9}$ Lysosomal membrane rupture and CatB activity are important for the activation of the NLRP3 inflammasome in response to some stimuli, where the inhibition of CatB attenuates IL-1 $\beta$ release ${ }^{10,11}$ Cathepsin $B$ also degrades collagens in fibroblasts and leads to tissue destruction. ${ }^{4,12}$

Proteasome is generally considered a dominating component in protein degradation pathway. ${ }^{13}$ Through degrading regulatory proteins or their inhibitors, proteasome regulates many cellular transduction pathways. The ubiquitin-proteasome pathway also offers extremely high substrate specificity and the ability to quickly alter the rate of proteolysis. ${ }^{13}$ Extracellular proteasomes have been found to circulate in the plasma of patients suffering from a variety of inflammatory, autoimmune and neoplastic diseases. ${ }^{14-21}$ In various pathologic conditions, the concentration of circulating proteasomes correlates with disease activity. ${ }^{14-21}$ The release of proteasome in the serum may be a result of membrane disruption. Endothelial cells, vascular smooth muscle cells and tubular epithelial cells all release proteasome-active vesicles when injured in vitro. ${ }^{14}$ A study by Ito et al. demonstrated that extracellular $20 \mathrm{~S}$ proteasome not only is released from cells, but also plays a functional role in physiological processes. ${ }^{13}$

Using the surface plasmon resonance imaging (SPRI) biosensor, we wanted to determine the concentration of $\mathrm{CatB}$ in the diagnostics of inflammatory condition located in the abdominal cavity and its correlation with $20 \mathrm{~S}$ proteasome.

\section{Material and methods}

Forty-two children with acute appendicitis, who were treated at the Department of Pediatric Surgery of Medical University of Białystok, Poland, between 2013 and 2014, were randomly included into the study (age 5-17 years, mean age $11.5 \pm 1$ year). There were 15 girls and 27 boys in the study group. Twenty-six children were subjected to laparoscopic appendectomy, while 16 children were subjected to classic open appendectomy. Eighteen healthy, agematched subjects, admitted for planned surgeries, served as controls. Exclusion criteria were: severe preexisting infections, immunological or cardiovascular diseases that required long-term medication, and complicated cases of appendicitis with perforation of the appendix and/or peritonitis. All parents of our patients gave written informed consent for both a clinical and biochemical followup. We did not note any postoperative complications in our patients after appendectomy. All patients were discharged home on the $3^{\text {rd }}$ day after surgery in good general state.

Venous blood samples (1-2 mL) were drawn on admission, $24 \mathrm{~h}$ after the appendectomy and $72 \mathrm{~h}$ after the appendectomy. Blood samples were collected in ethylenediaminetetraacetic acid (EDTA) tubes; the plasma was prepared according to standard protocols and was stored at $-80^{\circ} \mathrm{C}$. After all blood samples were collected and patient data recorded, the CatB and $20 \mathrm{~S}$ proteasome concentrations were assessed using SPRI by the investigators blinded to other data. ${ }^{22}$

\section{Procedure of cathepsin B and 205 proteasome determination}

The CatB and 20S proteasome concentration was determined using the SPRI biosensor. The exact description of the methodology of measurements and biosensor design was set out in previous papers. ${ }^{22}$ Gold chips were manufactured as described in other papers. ${ }^{23-25}$ The gold surface of the chip was covered with photopolymer and hydrophobic paint.

\section{Biosensor preparation}

Chips were rinsed with ethanol and water, and then dried under a stream of nitrogen. They were then immersed in $20 \mathrm{mM}$ of cysteamine ethanolic solutions for $2 \mathrm{~h}$ and, after rinsing with ethanol and water, dried again under a stream of nitrogen. ${ }^{16}$

\section{Surface plasmon resonance imaging measurements}

Surface plasmon resonance imaging for the protein biosensor array was performed as described in another study. ${ }^{23}$

Plasma samples from healthy children admitted for planned surgeries $(n=18)$ and from our patients after 
appendectomy $(\mathrm{n}=42)$ were diluted 2-fold with phosphatebuffered saline (PBS) (Biomed-Lublin, Lublin, Poland) and transferred onto the sensor surface for $10 \mathrm{~min}$. The volume of the sample applied on each measuring field was $2 \mu \mathrm{L}$. The SPRI technique allows sensitive determination of proteins using highly specific enzyme-inhibitor interactions. An immobilized cystatin C (potent inhibitor of lysosomal proteinases) was purchased from Sigma-Aldrich (St. Louis, USA) and used for the CatB entrapment on the biosensor surface. The biosensor construction and optimization of measurement conditions used were previously described. ${ }^{23}$ Briefly, plasma samples were placed directly on the prepared biosensor for $\sim 10 \mathrm{~min}$ to allow interaction with the inhibitor (cystatin C). The biosensor was washed with water and HBS-ES buffer solution, $\mathrm{pH}=7.4$ (0.01 M 4-(2-hydroxyethyl)piperazine-1-ethanesulfonic acid, $0.15 \mathrm{M}$ sodium chloride, $0.005 \%$ Tween 20,3 mM EDTA) (all Biomed-Lublin, Lublin, Poland) to remove unbound molecules from the surface. The SPRI signal was measured twice on the basis of registered images, following the immobilization of cystatin $\mathrm{C}$, and then following the interaction with $\mathrm{CatB}$ from the samples. The signal, which is proportional to coupled biomolecules, was obtained by calculating the difference between the signal prior to and following the interaction with biomolecules. The concentration was determined using the calibration curves of the SPRI signal depending on the concentration of CatB (Calbiochem; Merck, Warszawa, Poland).

\section{Statistics}

The Mann-Whitney U test and the Kruskal-Wallis H test with Dunn's post hoc correction to control for multiple testing were used to compare differences between groups. Statistical analyses were calculated with the STATISTICA PL
(StatSoft Inc., Tulsa, USA) v. 10.0 program. A two-tailed $\mathrm{p}<0.05$ was considered significant. Correlations were examined by linear regression ( $r$ ) using the Spearman's test. We presented median concentrations of CatB and 20S proteasome in children with appendicitis before surgery, as well as $24 \mathrm{~h}$ and $72 \mathrm{~h}$ after the appendectomy.

\section{Results}

The CatB concentrations in the blood plasma of patients with acute appendicitis were elevated before surgery, they were the highest $24 \mathrm{~h}$ after surgery, and were above the range of concentrations measured in controls, with the difference being statistically significant. The CatB concentrations measured $72 \mathrm{~h}$ after the operation were low, but still did not reach the normal range when compared with the concentrations measured in controls $(\mathrm{p}<0.05)$ (Fig. 1).

The $20 \mathrm{~S}$ proteasome concentrations in the blood plasma of patients with acute appendicitis were the highest before surgery, and were above the range of concentrations measured in controls, with the difference being statistically significant. The $20 \mathrm{~S}$ proteasome concentrations measured $24 \mathrm{~h}$ and $72 \mathrm{~h}$ after the operation slowly decreased over time, and still did not reach the normal range when compared with the concentrations measured in controls ( $<<0.05$ ) (Fig. 2). There was no statistical difference between the CatB and 20S proteasome concentrations in children operated on laparoscopically and in children after classic appendectomy (data not shown). There was a strong positive correlation between the CatB and $20 \mathrm{~S}$ proteasome concentrations $24 \mathrm{~h}$ after the surgical intervention $(\mathrm{r}=0.5871 ; \mathrm{p}=0.00004)$ (Fig. 3). Both proteasomal and lysosomal systems are responsible for proteolysis, which is more intensive under pathologic conditions, and,

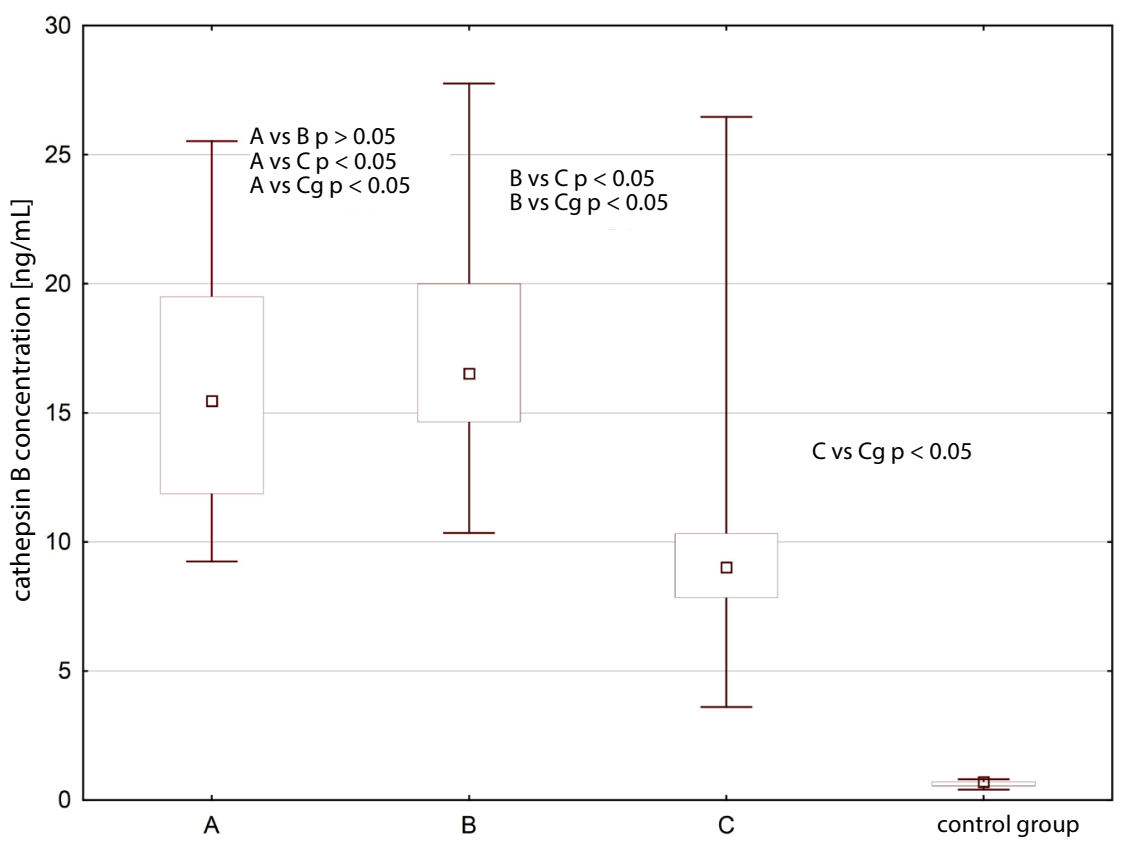

Fig. 1. Cathepsin $B$ concentration in the plasma of children with acute appendicitis before (A), $24 \mathrm{~h}$ after (B) and $72 \mathrm{~h}$ after (C) appendicitis surgery, and in control group $(\mathrm{Cg})$ 

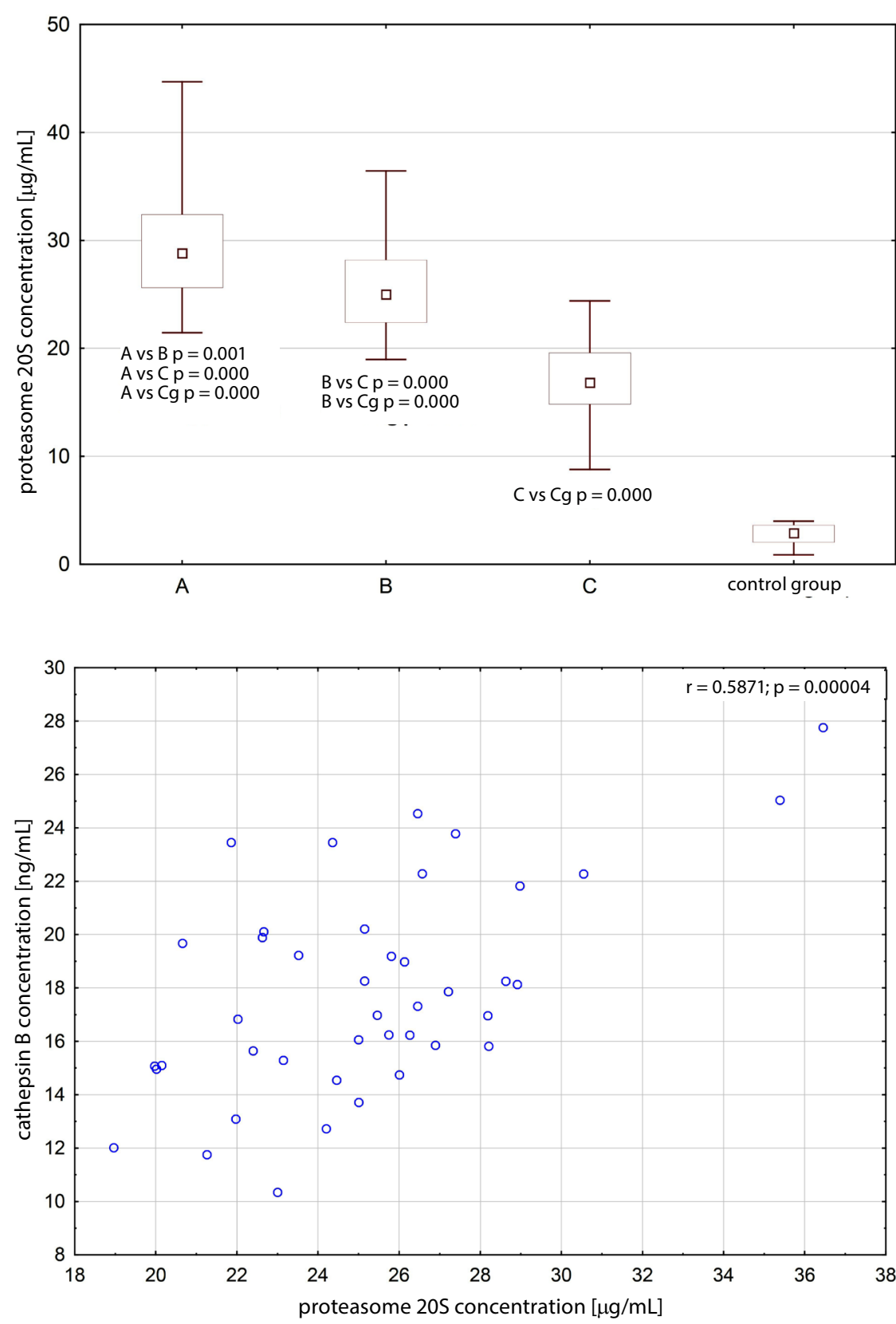

Fig. 2. Proteasome $20 \mathrm{~S}$ concentration in the plasma of children with acute appendicitis before (A), $24 \mathrm{~h}$ after (B) and $72 \mathrm{~h}$ after (C) appendicitis surgery, and in control group (Cg)

Fig. 3. Positive correlation between cathepsin $B$ and 205 proteasome concentrations in the blood of children $24 \mathrm{~h}$ after appendectomy in this case, was induced by inflammation and enhanced by surgical intervention. There were no such correlations before and $72 \mathrm{~h}$ after the surgery $(\mathrm{p}>0.05)$.

\section{Discussion}

Appendicitis is caused by a blockage of the hollow portion of the appendix, most commonly by a calcified "stone" made of feces, but also by inflamed lymphoid tissue from a viral infection, parasites or tumors. This blockage leads to increased pressure in the lumen of the appendix, decreased blood flow in the appendiceal wall and bacterial growth inside the appendix, causing inflammation. The combination of inflammation, reduced blood flow to the appendix and distention of the appendix causes tissue injury and tissue death.
Protein degradation occurs in response to the activation of intracellular signaling pathways that increase the activity of the ubiquitin-proteasome, calcium-induced calpains and apoptotic regulators, such as caspase- $3 .^{27,28}$ Various pathologic stresses induce the release of cathepsins - acidic endopeptidases, the activity of which is restricted to the lysosomal compartment - into the cytoplasm, where they perform their proteolytic function and can promote direct cell degradation. ${ }^{1,3}$

Cathepsin B is released from lysosomes after the activation of specific apoptotic inducers, such as death receptors of the TNF family, transforming growth factor-beta 1 (TGF- $\beta 1$ ), p53, and sphingosine, as well as during oxidative stress and starvation. Sphingosine induces lysosomal permeabilization in a CatB-dependent manner. ${ }^{1,5}$

In our study, we found that the CatB concentrations in the blood plasma of patients with acute appendicitis were 
elevated before surgery, they were the highest $24 \mathrm{~h}$ after surgery, and $72 \mathrm{~h}$ after the surgical intervention they were still above the range of concentrations measured in controls. This reflects the metabolic response to acute state of inflammation, surgical intervention and the process of gradual ebbing of the inflammation. We can conclude that lysosomal proteolysis connected with CatB activity was induced by inflammation and exacerbated by surgical intervention. Positive correlation between the CatB and proteasome concentrations $24 \mathrm{~h}$ after surgical intervention indicates higher destruction of cells and higher release of their content into the blood.

Apparently, the method of operation - classic open appendectomy or laparoscopic appendectomy - did not influence the general trend in the CatB and $20 \mathrm{~S}$ proteasome concentrations in the blood of children with appendicitis. We may hypothesize that the resection of the appendix itself, regardless of the method of the operation, is the most influential factor in the process of reducing the inflammation resulting from acute appendicitis.

Cathepsin B regulates collagen expression by fibroblasts via prolonging toll-like receptor 2 (TLR2)/NF- $\mathrm{KB}$ activation during chronic inflammation and oxidative stress. ${ }^{4}$ It is involved in degrading the intracellular and extracellular collagen produced by fibroblasts, thereby influencing the mechanisms of delayed tissue repair during chronic inflammation. Collagen type III and type IV in fibroblasts are important in wound healing and tissue remodeling. ${ }^{4}$ Considering the role of CatB in collagen expression, CatBspecific inhibitors may be a useful approach for improving inflammation-delayed connective tissue repair, such as that found in dermatitis and periodontitis. ${ }^{4}$

Cathepsin B was also hypothesized to be involved in the generation of chronic pain. ${ }^{29}$ It was demonstrated to be involved in the production of IL-1 $\beta$, which is a key painrelated molecule. ${ }^{29}$ The role of CatB in inflammatory pain suggests that $\mathrm{CatB}$-specific inhibitors may represent a useful new strategy for treating inflammation-associated pain, such as arthritic pain and postoperative pain. ${ }^{29}$

Cathepsin B expression is greatly upregulated in traumatic brain injury (TBI) animal models, as well as in trauma patients. Knockout of the CatB gene in TBI mice results in substantial improvements of TBI-caused deficits in behavior, pathology and biomarkers, as well as improvements in related injury models. ${ }^{30}$ During the process of TBI-induced injury, CatB likely escapes the lysosome, its normal subcellular location, into the cytoplasm or extracellular space. Cathepsin B in the extracellular space can induce neuronal cell apoptotic death. Non-brain polytrauma patients show increases in plasma CatB activity during the $1^{\text {st }}$ day after trauma, which subsequently falls to moderately elevated levels by the $3^{\text {rd }}$ day and remains roughly at that level for up to 2 weeks. ${ }^{30}$ Importantly, the increase in plasma CatB activity correlates with the severity of injury. ${ }^{30-32}$ Deleting or inhibiting CatB improves outcomes in injury models related to TBI, including epilepsy, aneurysm, ischemia, pain, surgical trauma, spinal cord trauma, infectious disease, and neurodegeneration. ${ }^{30}$

Cathepsin B is also a candidate target for inhibiting hepatocyte apoptosis in liver diseases. ${ }^{33}$ Both genetic and pharmacologic inactivation of CatB reduces liver injury, inflammation and hepatic fibrogenesis during cholestasis. ${ }^{24}$ Cathepsin B-mediated liver injury not only causes apoptosis, but also stimulates the production of proinflammatory chemokines. ${ }^{33}$ Furthermore, inhibiting CatB catalytic activity with selective protease inhibitors, such as R-3032, might be a potential therapeutic option for cholestatic liver injury, inflammation and fibrosis. ${ }^{33}$

Cathepsin B is a potential drug target for several diseases, including various cancers, pancreatitis, liver fibrosis, rheumatoid arthritis, viral Ebola, bacterial Streptococcus pneumoniae meningitis, TBI, and parasitic Trypanosoma cruzi infections. ${ }^{4,30,34-43}$ We strongly believe that further studies on CatB and its relation with inflammation located in the abdominal cavity and surgical interventions may bring future clinical implications.

\section{Conclusions}

Cathepsin B concentration may reflect the metabolic response to acute state of inflammation, surgical intervention in the abdominal cavity and the process of gradual ebbing of inflammation. The method of operation - classic open appendectomy or laparoscopic appendectomy - does not influence the general trend in the $\mathrm{CatB}$ concentration in children with appendicitis. There is a strong positive correlation between the CatB and $20 \mathrm{~S}$ proteasome concentrations $24 \mathrm{~h}$ after surgery. Biosensors with SPRI as a detecting method can be successfully used for determining the concentration of active forms of enzymes presented in lysosomes in the diagnostics of inflammatory condition in the abdominal cavity.

\section{References}

1. Benchoua A, Braudeau J, Reis A, Couriaud C, Onténiente B. Activation of proinflammatory caspases by cathepsin $B$ in focal cerebral ischemia. J Cereb Blood Flow Metab. 2004;24(11):1272-1279.

2. Uchiyama Y. Autophagic cell death and its execution by lysosomal cathepsins. Arch Histol Cytol. 2001;64(3):233-246.

3. Boya P, Andreau K, Poncet D, et al. Lysosomal membrane permeabilization induces cell death in a mitochondrion-dependent fashion. J Exp Med. 2003;197(10):1323-1334.

4. Li X, Wu Z, Ni J, et al. Cathepsin B regulates collagen expression by fibroblasts via prolonging TLR2/NF-KB activation. Oxid Med Cell Longev. 2016:7894247. doi: 10.1155/2016/7894247

5. Foghsgaard L, Wissing D, Mauch D, et al. Cathepsin B acts as a dominant execution protease in tumor cell apoptosis induced by tumor necrosis factor. J Cell Biol. 2001;153(5):999-1010.

6. Terada K, Yamada J, Hayashi Y, et al. Involvement of cathepsin B in the processing and secretion of interleukin-1 $\beta$ in chromogranin a-stimulated microglia. Glia. 2010;58(1):114-124.

7. Sun L, Wu Z, Hayashi Y, et al. Microglial cathepsin B contributes to the initiation of peripheral inflammation-induced chronic pain. J Neurosci. 2012;32(33):11330-11342.

8. Wu Z, Sun L, Hashioka S, et al. Differential pathways for interleukin-1 $\beta$ production activated by chromogranin $A$ and amyloid $\beta$ in microglia. Neurobiol Aging. 2013;34(12):2715-2725. 
9. Dinarello CA. Biologic basis for interleukin-1 in disease. Blood. 1996; 87(6):2095-2147.

10. Hornung V, Bauernfeind F, Halle A, Samstad EO, Kono H, Rock KL. Silica crystals and aluminum salts activate the NALP3 inflammasome through phagosomal destabilization. Nat Immunol.2008;9(8):847-856.

11. Halle A, Hornung V, Petzold GC, Stewart CR, Monks BG, Reinheckel T. The NALP3 inflammasome is involved in the innate immune response to amyloid-beta. Nat Immunol. 2008;9(8):857-865.

12. Geraghty $P$, Rogan MP, Greene $C M$, et al. Neutrophil elastase up-regulates cathepsin B and matrix metalloprotease-2 expression. J/mmunol. 2007;178(9):5871-5878.

13. Ito WD, Lund N, Zhang Z, et al. Activation of cell surface bound $20 \mathrm{~S}$ proteasome inhibits vascular cell growth and arteriogenesis. Biomed Res Int. 2015:719316. doi: 10.1155/2015/719316

14. Dieudé $M$, Bell C, Turgeon J, et al. The 20 S proteasome core, active within apoptotic exosome-like vesicles, induces autoantibody production and accelerates rejection. Sci Trans/Med. 2015;7(318):318-200. doi: 10.1126/scitranslmed.aac9816

15. Majetschak M, Perez M, Sorell LT, Lam J, Maldonado ME, Hoffman RW. Circulating $20 \mathrm{~S}$ proteasome levels in patients with mixed connective tissue disease and systemic lupus erythematosus. Clin Vaccine Immunol. 2008;15(9):1489-1493.

16. Henry L, Lavabre-Bertrand T, Douche T, et al. Diagnostic value and prognostic significance of plasmatic proteasome level in patients with melanoma. Exp Dermatol. 2010;19:1054-1059.

17. Jakob C, Egerer K, Liebisch $P$, et al. Circulating proteasome levels are an independent prognostic factor for survival in multiple myeloma. Blood. 2007;109(5):2100-2105.

18. Matuszczak E, Tylicka M, Dębek W, Hermanowicz A, Ostrowska H. The comparison of C-proteasome activity in the plasma of children after burn injury, mild head injury and blunt abdominal trauma. Adv Med Sci. 2015;60(2):253-258.

19. Tylicka M, Matuszczak E, Dębek W, Hermanowicz A, Ostrowska H. Circulating proteasome activity following mild head injury in children. Childs Nerv Syst. 2014;30(7):1191-1196.

20. Matuszczak E, Tylicka M, Dębek W, Hermanowicz A, Ostrowska H. Correlation between circulating proteasome activity, total protein and c-reactive protein levels following burn in children. Burns. 2014;40(5): 842-847.

21. Sixt SU, Dahlmann B. Extracellular, circulating proteasomes and ubiquitin - incidence and relevance. Biochim Biophys Acta. 2008;1782(12): 817-823.

22. Sankiewicz A, Laudański P, Romanowicz L, et al. Development of surface plasmon resonance imaging biosensors for detection of ubiquitin carboxyl-terminal hydrolase L1. Anal Biochem. 2015;469:4-11.

23. Gorodkiewicz E. Surface plasmon resonance imaging sensor for cathepsin determination based in immobilized cystatin. Protein Pept Lett. 2009;16(11):1379-1385.

24. Gorodkiewicz E, Sieńczyk M, Regulska E, et al. Surface plasmon resonance imaging biosensor for cathepsin $\mathrm{G}$ based on a potent inhibitor: Development and applications. Anal Bochem. 2012;423:218-223.

25. Gorodkiewicz E, Sankiewicz A, Laudański P. Surface plasmon resonance imaging biosensors for aromatase based on a potent inhibitor and a specific antibody: Sensor development and application for biological material. Cent Eur J Chem. 2014;12(5):557-567.
26. Johnsson B, Lofas S, Lindquist G. Immobilization of proteins to a carboxymethyldextran-modified gold surface for biospecific interaction analysis in surface plasmon resonance sensors. Anal Biochem. 1991;198:268-277.

27. Jeschke MG, Gauglitz GG, Kulp GA, et al. Long-term persistance of the pathophysiologic response to severe burn injury. PLoS ONE. 2011;6(7): e21245. doi: 10.1371/journal.pone.0021245

28. Porter C, Herndon DN, Sidossis LS, et al. The impact of severe burns on skeletal muscle mitochondrial function. Burns. 2013;39(6):1039-1047.

29. Sun L, Wu Z, Hayashi Y, et al. Microglial cathepsin B contributes to the initiation of peripheral inflammation-induced chronic pain. J Neurosci. 2012;32(33):11330-11342.

30. Hook G, Jacobsen JS, Grabstein K, Kindy M, Hook V. Cathepsin B is a new drug target for traumatic brain injury therapeutics: Evidence for E64d as a promising lead drug candidate. Front Neurol. 2015;6:178. doi: 10.3389/fneur.2015.00178

31. Assfalg-Machleidt I, Jochum M, Nast-Kolb D, et al. Cathepsin B-indicator for the release of lysosomal cysteine proteinases in severe trauma and inflammation. Biol Chem Hoppe Seyler. 1990;371(Suppl): 211-222.

32. Jochum M, Machleidt W, Fritz H. Phagocyte proteinases in multiple trauma and sepsis: Pathomechanisms and related therapeutic approaches. In: Neugebauer EA, Holaday JW, eds. Handbook of Mediators in Septic Shock. Boca Raton, FL: CRC Press; 1993:335-361.

33. Canbay A, Guicciardi ME, Higuchi $H$, et al. Cathepsin B inactivation attenuates hepatic injury and fibrosis during cholestasis. JClin Invest. 2003;112(2):152-159.

34. Gondi CS, Rao JS. Cathepsin B as a cancer target. Expert Opin Ther Targets. 2013;17(3):281-291.

35. Palermo C, Joyce JA. Cysteine cathepsin proteases as pharmacological targets in cancer. Trends Pharmacol Sci. 2008;29(1):22-28.

36. Kos J, Mitrovic A, Mirkovic B. The current stage of cathepsin B inhibitors as potential anticancer agents. Future Med Chem. 2014;6(11): 1355-1371.

37. Cha IS, Kwon J, Mun JY, et al. Cathepsins in the kidney of olive flounder, Paralichthys olivaceus, and their responses to bacterial infection. Dev Comp Immunol. 2012;38(4):538-544.

38. Canbay A, Feldstein AE, Higuchi $H$, et al. Kupffer cell engulfment of apoptotic bodies stimulates death ligand and cytokine expression. Hepatology. 2003;38(5):1188-1198.

39. Cunnane G, Fitzgerald O, Beeton C, Cawston TE, Bresnihan B. Early joint erosions and serum levels of matrix metalloproteinase 1, matrix metalloproteinase 3, and tissue inhibitor of metalloproteinases 1 in rheumatoid arthritis. Arthritis Rheum. 2001;44(10):2263-2274.

40. Mishiro T, Nakano S, Takahara S, et al. Relationship between cathepsin B and thrombin in rheumatoid arthritis. J Rheumatol. 2004;31(7): 1265-1273.

41. Baici A, Müntener K, Willimann A, Zwicky R. Regulation of human cathepsin $B$ by alternative mRNA splicing: Homeostasis, fatal errors and cell death. Biol Chem. 2006;387(8):1017-1021.

42. Feng $Y, N i$ L, Wang Q. Administration of cathepsin B inhibitor CA-074Me reduces inflammation and apoptosis in polymyositis. J Dermatol Sci. 2013;72(2):158-167.

43. Bien S, Ritter CA, Gratz M, et al. Nuclear factor-кB mediates up-regulation of cathepsin B by doxorubicin in tumor cells. Mol Pharmacol. 2004;65(5):1092-1102. 